\title{
Brazilian Psychiatric Association guidelines for the management of suicidal behavior. Part 2. Screening, intervention, and prevention
}

\author{
Leonardo Baldaçara, ${ }^{1,2}$ (iD Roberta R. Grudtner, ${ }^{2,3}$ (iD Verônica da S. Leite, ${ }^{1,4}$ (iD Deisy M. Porto, ${ }^{2,5}$ (iD \\ Kelly P. Robis, ${ }^{6,7}$ iD Thiago M. Fidalgo, ${ }^{8}$ (iD Gislene A. Rocha, ${ }^{2,9}$ iD Alexandre P. Diaz, ${ }^{10,11}$ iD \\ Alexandrina Meleiro, ${ }^{2}$ (iD Humberto Correa, ${ }^{2,7}$ (iD Teng C. Tung, ${ }^{2,12}$ (iD Leandro Malloy-Diniz, ${ }^{7,13}$ (iD \\ João Quevedo, ${ }^{10,14}$ (iD) Antônio G. da Silva ${ }^{2,15}$ (iD

\begin{abstract}
${ }^{1}$ Universidade Federal do Tocantins (UFT), Palmas, TO, Brazil. ${ }^{2}$ Associação Brasileira de Psiquiatria (ABP), Rio de Janeiro, RJ, Brazil. Município de Palmas, Palmas, TO, Brazil. ${ }^{5}$ Associação Catarinense de Psiquiatria, Florianópolis, SC, Brazil. ${ }^{6}$ Departamento de Psiquiatria, Pontifícia Universidade Católica de Minas Gerais (PUC Minas), Belo Horizonte, MG, Brazil. ${ }^{7}$ Departamento de Psiquiatria, Universidade Federal de Minas Gerais (UFMG), Belo Horizonte, MG, Brazil. ${ }^{8}$ Departamento de Psiquiatria, Universidade Federal de São Paulo (UNIFESP), São Paulo, SP, Brazil. ${ }^{9}$ Hospital Universitário Clemente de Faria, Montes Claros, MG, Brazil. ${ }^{10}$ Translational Psychiatry Program, Faillace Department of Psychiatry and Behavioral Sciences, McGovern Medical School, The University of Texas Health Science Center at Houston (UTHealth), Houston, TX, USA. ${ }^{11}$ Laboratório de Psiquiatria Translacional, Programa de Pós-Graduação em Ciências da Saúde, Universidade do Extremo Sul Catarinense (UNESC), Criciúma, SC, Brazil. ${ }^{12}$ Instituto de Psiquiatria, Hospital das Clínicas, Faculdade de Medicina, Universidade de São Paulo (USP), São Paulo, SP, Brazil. ${ }^{13}$ Associação Brasileira de Impulsividade e Patologia Dual, Brasília, DF, Brazil. ${ }^{14}$ Center of Excellence on Mood Disorders, Faillace Department of Psychiatry and Behavioral Sciences, McGovern Medical School, The
\end{abstract} \\ ${ }^{3}$ Hospital Psiquiátrico São Pedro, Secretaria Estadual da Saúde do Rio Grande do Sul, Porto Alegre, RS, Brazil. ${ }^{4}$ Secretaria de Saúde do \\ University of Texas Health Science Center at Houston (UTHealth), Houston, TX, USA. ${ }^{15}$ Asociación Psiquiátrica de América Latina (APAL).
}

\begin{abstract}
This article continues our presentation of the Brazilian Psychiatric Association guidelines for the management of patients with suicidal behavior, with a focus on screening, intervention, postvention, prevention, and promotion. For the development of these guidelines, we conducted a systematic review of the MEDLINE (via PubMed), Cochrane Database of Systematic Reviews, Web of Science, and SciELO databases for research published from 1997 to 2020. Systematic reviews, clinical trials, and cohort/observational studies on screening, intervention, and prevention in suicidal behavior were included. This project involved 14 Brazilian psychiatry professionals and 1 psychologist selected by the Psychiatric Emergencies Committee of the Brazilian Psychiatric Association for their experience and knowledge in psychiatry and psychiatric emergencies. Publications were evaluated according to the 2011 Oxford Center for Evidence-Based Medicine (OCEBM) Levels of Evidence Classification. Eightyfive articles were reviewed (of 5,362 initially collected and 755 abstracts on the drug approach). Forms of screening, intervention, and prevention are presented. The intervention section presents evidence for psychotherapeutic and drug interventions. For the latter, it is important to remember that each medication is effective only for specific groups and should not replace treatment protocols. We maintain our recommendation for the use of universal screening plus intervention. Although the various studies differ in terms of the populations evaluated and several proposals are presented, there is already significant evidence for certain interventions. Suicidal behavior can be analyzed by evidence-based medicine protocols. Currently, the best strategy is to combine several techniques through the Safety Plan. Nevertheless, further research on the topic is needed to elucidate some approaches with particular potential for intervention and prevention.
\end{abstract}

Systematic review registry number: CRD42020206517

Keywords: Suicide; completed suicide; suicide attempt; mental disorders; guideline

\section{Introduction}

In recent decades, suicide has grown considerably in incidence to become one of the leading causes of death

Correspondence: Leonardo Baldaçara, Quadra 401 Sul, Av. Joaquim Teotônio Segurado, 1, Espaço Médico, sala 1006, CEP 77015-550, Palmas, TO, Brazil.

E-mail: leonardobaldassara@gmail.com

Submitted Jun 06 2020, accepted Sep 10 2020, Epub Dec 092020. worldwide. ${ }^{1,2}$ Approximately 800,000 suicides are completed worldwide each year, which means that every 40 seconds, a person dies by suicide. ${ }^{1}$ In 2016 , Brazil had 9.7 deaths by suicide per 100,000 population, which

How to cite this article: Baldaçara L, Grudtner RR, Leite VS, Porto DM, Robis KP, Fidalgo TM, et al. Brazilian Psychiatric Association guidelines for the management of suicidal behavior. Part 2. Screening, intervention, and prevention. Braz J Psychiatry. 2021;43:538549. http://dx.doi.org/10.1590/1516-4446-2020-1108 
generates great concern, even though this number seems small when compared to the numbers of other countries in America. ${ }^{1,2}$

After years of research, it is already possible to identify the effectiveness of interventions and prevention based on the survey of risk factors and clinical research. ${ }^{3}$ Although the evidence is still unsatisfactory and controversies remain, some procedures with definite impact on specific patient populations have already been identified.

Within this context, the Brazilian Psychiatric Association has developed a series of guidelines for the management of patients with suicidal behavior. The first part of the guidelines, concerning risk factors, protective factors, and assessment, has been published in the Journal. ${ }^{4}$ This article now presents the second part, with an emphasis on screening, intervention, prevention, and promotion.

\section{Method}

This project involved 14 Brazilian professionals (13 psychiatrists and 1 psychologist) selected by the Psychiatric Emergencies Committee (Comissão de Emergências Psiquiátricas) of the Brazilian Psychiatric Association for their experience and knowledge in psychiatry and psychiatric emergencies. For the development of these guidelines, the MEDLINE (via PubMed), Cochrane Database of Systematic Review, Web of Science, and SciELO databases were searched for articles published from 1997 to 2020, in English or Portuguese. The search strategy used was based on questions structured according to the PICO format ("patient or population," "intervention or exposure," "control or comparison," and "outcome"), as recommended by the Guidelines Project of the Brazilian Medical Association (AMB). The use of structured clinical questions aimed at facilitating the elaboration of strategies to search for evidence. The descriptors used were "suicide" AND "screening" OR "intervention" OR "prevention" OR "promotion." Systematic reviews with meta-analysis were prioritized, and other types of research were sought only when the information was not found.

When analyzing the literature, even with a large number of clinical trials and reviews, some difficulties were found in the evaluation of results: the evaluation of suicidal behavior with several different diagnoses; the assessment and monitoring of suicidal behavior in different contexts, and with different criteria and instruments; certain studies analyzed interventions in a small number of patients. Therefore, the following criteria were implemented: 1) studies on suicidal behavior for adolescents, adults, and the elderly; and 2) objective assessment of response, either by reduction in symptoms or by an objective scale. The exclusion criteria were as follows: 1) studies with fewer than 20 participants in the sample; and 2 ) incomplete data and low-quality statistical analysis.

In addition, articles deemed relevant to the literature were also used in the development of this guideline. The selection process for such articles proceeded as followed: (I) selection of the relevant article abstracts; (II) reading the relevant articles in full; (III) critical analysis of the evidence; and (IV) extraction of results and classification of evidence strength. Levels of evidence and grades of recommendation were selected in accordance with the 2011 Oxford classification. (For further information, see https://www.cebm.net/wp-content/uploads/2014/06/

CEBM-Levels-of-Evidence-2.1.pdf.)

Of 5,362 entries and 755 abstracts initially retrieved, 85 articles were ultimately reviewed. Figure 1 shows the study selection flowchart, designed according to the Preferred Reporting Items for Systematic Reviews and Meta-Analyses (PRISMA Statement). Table S1, available as online-only supplementary material, provides details as well as the level of evidence of the selected articles.

\section{Screening}

When an instrument or protocol is used to distinguish individuals with suicidal behavior, this is called screening. Screening for suicidality can be done independently or as part of a broader health screen. It can be done by taking notes on paper or with the aid of electronic devices, such as tablets or computers, or even with the examiner asking questions orally; and it can be applied selectively or universally (applied to everyone in the target population, e.g., all students of a school, regardless of whether they are at greater risk than healthy individuals). ${ }^{6}$

Suicide assessment must be carried out by a physician, to identify the immediate risk for the patient and define the course of the intervention. Assessment may involve structured questionnaires; however, it must also include a more open-ended conversation with the patient, family, or friends to obtain information about the patient's behavior, risk and protective factors, and previous history of medical and mental health care. Another useful aspect of assessment is to guide development of individualized intervention plans and monitor their progress. ${ }^{6}$

However, as already discussed in the previous part of these guidelines, there is no single factor capable of predicting suicidal behavior. Existing instruments also demonstrate limited effectiveness; therefore, assessment must be wide-ranging ${ }^{7}$ (Level of evidence 3 ), with instruments used as a supplemental tool within the assessment. Considering the complexity of this assessment and that it is not possible to carry it out on a large scale, we suggest that any sign or symptom that a person indicates the desire, thought, or attempt to injure themselves or end their life be used as screening.

The general prevalence of suicidal communication (SC) prior to suicide is $44.5 \%$ (although there is substantial publication bias). ${ }^{8}$ The prevalence of SC varies according to different studies, including with regard to their methodological quality. ${ }^{8} \mathrm{SC}$ was related to an OR of 4.66 for suicide, with satisfactory diagnostic accuracy only when studies in adolescents were withdrawn, based on seven case-control studies. ${ }^{8}$ The evidence suggests that family members, law enforcement, and health professionals should refer any individuals who communicate suicidal intent to appropriate mental health care (Level of evidence 3).

In part 1 of these guidelines, we suggested that a thorough assessment is even more effective if it is combined with intervention strategies, as noted in previous research.,9-11 Then, we suggested, under the name of the Safety Plan, a form of approach that unites, as in 


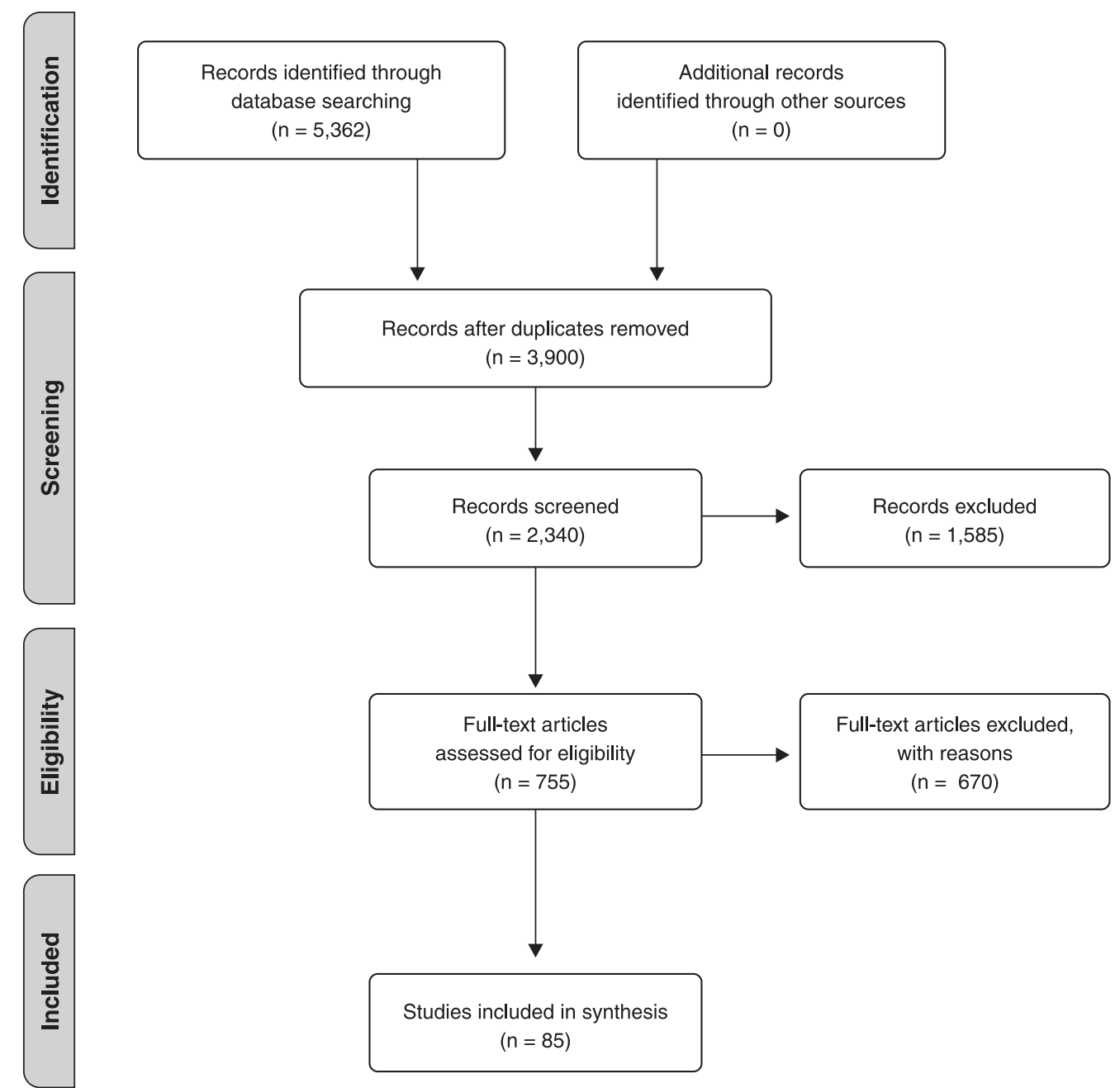

Figure 1 PRISMA flowchart for the study selection process ${ }^{5}$.

these works, the assessment, including risk and protection factors, already in sequence with therapeutic plan.

\section{Intervention}

Follow-up

Patients at risk of suicide, especially those who have attempted suicide, need follow-up and supervision to ensure adequate outcomes. Surveillance should begin while the patient is still in the hospital environment. Patients who leave the emergency room or hospital after a suicide attempt or who have an imminent and significant risk of suicide identified at any health facility need immediate and proactive follow-up. The frequency of contact should be determined individually and intensified when there are increases in risk factors. Strengthening the Safety Plan at regular intervals, including additional practices and, if necessary, revisions, are important factors in a supportive approach. ${ }^{12}$

A meta-analysis conducted by Malone et al. ${ }^{13}$ analyzed the effects of care provided by a community mental health team $(\mathrm{CMHT})$ to people with severe mental illness. Data were collected with the assistance of a nonstandardized team. Although hospitalization rates were significantly lower in the CMHT group, contact with primary care, hospitalization in emergency units for trauma care, and contact with social services was not significantly different between the compared groups. Although fewer deaths occurred in the CMHT group, management by the CMHT was not associated with significant changes in deaths by suicide or deaths in suspicious circumstances. ${ }^{13}$

In addition, in another meta-analysis, intensive case management (ICM), a community help service that aims to provide long-term care to patients with severe mental disorders, has been shown to make little or no difference in reducing death by suicide. The results of this study did not provide scientific evidence that additional psychosocial interventions after personal injuries have a marked effect on reducing the risk of suicide. ${ }^{14,15}$

In one before-and-after study, a general practitioner (GP)-based intervention was observed. Indicators of a treatment effect for GP training on suicide mortality rates were not evident. This evidence was also not found in 
most of the other results studied. ${ }^{16}$ These data suggest that training alone is not enough for generalists to manage cases of suicidal behavior, and that presence of a specialist is always recommended.

In addition, there is no significant efficacy of brief contact interventions for recurrent self-inflicted injuries, suicide, or attempted suicide, nor is there a relevant impact on the occurrence of self-inflicted injuries or recurrent suicide attempts per person (only three studies served as the basis). ${ }^{17}$ The number of people who attempted suicide again and the number of suicide deaths during the following year were not reduced by telephone contact. ${ }^{18}$

As previously discussed, screening, evaluation, and intervention are more effective if done together through universal screening plus intervention or a Safety Plan ${ }^{9,11}$ (Level of evidence 3). Follow-up must necessarily be included. Box 1 provides recommendations for follow-up according to the modified Safety Plan as suggested by author Boudreaux et al. ${ }^{9}$

Surveillance should be intensified (attempting home care) or the individual should be referred immediately to the emergency department (ED) or hospital in the case of the following:

- Various risk factors associated with a lack of family support;

- Various risk factors associated with a lack of access to outpatient health services;

- Recent and frequent suicide attempts;

- Verbalization of a suicide plan;

- Suicidal behavior associated with reward-based illness (such as substance abuse), psychotic breakdown, manic state, or worsening depression despite treatment;

- Change in the status of the observation or the intervention scenario (for example, discharge from the hospital environment);
- Gradual worsening or no improvement, despite treatment.

The patient should always be taken to the ED facility or hospital by ambulance and approached by trained staff, such as emergency medical technicians or paramedics.

\section{In the emergency department (ED) or hospital}

The patient cannot be left alone. Bathrooms should not have doors that can be locked. A multidisciplinary approach should be available, especially daily meetings with a psychiatrist and psychotherapeutic intervention by a psychologist or psychiatrist. The environment must be free of any object or substance that patients may use to attempt to take their own life. Great care must be taken on upper floors (windows and exits must be protected), and cleaning products must not be easily accessible to the patient. The possibility of discharge should be considered only when the patient has improved, and access to followup in the community and the possibility that a family member or trusted companion can continue supervision should be considered.

\section{Managing intoxicated and suicidal patients}

In cases highly suggestive of attempted suicide through intoxication by alcohol or other drugs, as demonstrated by the clinical presentation or objective data (breath or laboratory tests, for example), the patient must remain in a safe environment until there is certainty that intoxication has ceased. Later, a reassessment of the risk of suicide must be performed. ${ }^{12}$

In addition, a brief therapeutic intervention can be offered once the intoxication has worn off. In fact, the moment when an individual presents to the service due to suicidal behavior, regardless of whether the moment is of

Box 1 Recommendations for follow-up within the Safety Plan*

\begin{tabular}{|c|c|}
\hline Time point & Goals \\
\hline Week 1 & $\begin{array}{l}\text { - Explain the program and establish rapport } \\
\text { - Obtain the history (psychiatric and suicidal behavior) } \\
\text { - Assess status (suicide risk, psychiatric, caregivers) } \\
\text { - If necessary, develop/review the Safety Plan } \\
\text { - Work on building values and starting a life based on values }\end{array}$ \\
\hline Week 2 & $\begin{array}{l}\text { - Assess status (suicide risk, raise psych symptoms, and identify treatment providers and adherence) } \\
\text { - If necessary, review the Safety Plan } \\
\text { - Continue discussion of a life based on values } \\
\text { - Help to develop a "Life Plan" to monitor and reduce or eliminate the potential risk factors identified } \\
\text { - Emphasize the importance of collaborative monitoring and problem solving with significant others }\end{array}$ \\
\hline Week 3 & $\begin{array}{l}\text { - Explain the program and establish a relationship } \\
\text { - Raise other concerns regarding the patient } \\
\text { - Perform brief psychoeducation in relation to suicide and risk factors } \\
\text { - Present the life/Safety Plan to others }\end{array}$ \\
\hline Weeks $4,10,22,34$ and 48 & $\begin{array}{l}\text { - Assess the risk of suicide and the presence of psychiatric symptoms } \\
\text { - Review the Safety Plan and treatment adherence } \\
\text { - Discuss/informally problem solve identified issues/risk factors }\end{array}$ \\
\hline Weeks 8,20 and 32 & $\begin{array}{l}\text { - Review other significant patient concerns and perceptions } \\
\text { - Review the Safety Plan and treatment adherence } \\
\text { - Discuss/informally problem-solve identified issues/risk factors }\end{array}$ \\
\hline
\end{tabular}

\footnotetext{
* Modified from Boudreaux et al. ${ }^{9}$
} 
short duration, can represent an opportunity for reflection and learning, during which the individual may be more susceptible to having a discussion about the Safety Plan and other specific interventions. A Safety Plan should include the elimination of access to firearms as well as access to other means of suicide, including the restriction of access to sharp objects, hoses, ropes, or wires, and assurances of the safe storage or distribution of medicines as a high priority. ${ }^{12}$

It is also necessary to ensure that suicide risk management is integrated with treatment for substanceuse disorders and any other comorbidities, including communication with the patient's primary health care provider. ${ }^{12}$ When dealing with intoxicated patients, physicians should be aware that suicidal ideation or other behaviors do not typically result from acute substance use, and should consider such individuals as being at high risk of suicide. Accordingly, detoxification alone is insufficient in the presence of suicidal behaviors. ${ }^{12}$

\section{Adolescents}

The Saving and Empowering Young Lives in Europe (SEYLE) study is a multicenter, cluster-randomized controlled trial which compared interventions for suicidal behavior in 11,110 adolescent pupils (median age 15 years, IQR 14-15) recruited from 168 schools across 10 European Union countries. ${ }^{19}$ In a 3-month follow-up of adolescents with suicidal behavior, there was no significant difference between individuals who received the intervention and those who did not. At 12-month followup, the Conscious Mental Health for Young People (YAM) program achieved a greater reduction in suicidal ideation and suicide attempts compared to the control group ${ }^{19}$ (Level of evidence 2).

YAM is a universal intervention manual with a focus on students that includes 3 hours of interactive workshops associated with roleplaying and take-home material, consisting of six educational posters that are displayed in each educational environment (classrooms) as well as a 1-hour lecture on mental health, divided into two interactive lectures, shown before and after the intervention. ${ }^{19}$ YAM aimed to increase understanding about mental health and protective/risk factors related to suicide and depression and anxiety and to implement actions that improve the ability of individuals to deal with adverse life events, suicidal behavior, and stress in general. ${ }^{19}$

\section{Psychotherapy}

Suggested psychotherapies for suicidal behavior management and their level of evidence are presented in Table 1.

First, it is important to note that some negative results of psychosocial and psychotherapy interviews are possible. For example, the completion of a meta-analysis does

Table 1 Suggested psychotherapies for suicidal behavior management

\begin{tabular}{|c|c|c|c|}
\hline Type & Indication & $\begin{array}{l}\text { Level of } \\
\text { evidence }\end{array}$ & Warning \\
\hline Psychotherapy ${ }^{20}$ & $\begin{array}{l}\text { Patients with bipolar disorder, patients with } \\
\text { depression, patients with schizophrenia- } \\
\text { spectrum disorders }{ }^{20}\end{array}$ & 3 & \\
\hline $\begin{array}{l}\text { Brief psychodynamic interpersonal } \\
\text { psychotherapy }\end{array}$ & Borderline personality disorder ${ }^{12}$ & 5 & \\
\hline \multirow[t]{2}{*}{ Cognitive-behavioral therapy } & - Adolescents ${ }^{21}$ & 3 & \multirow[t]{2}{*}{$\begin{array}{l}\text { Face-to-face only. e-health was not } \\
\text { found to be efficacious for } \\
\text { reducing suicidal ideation and } \\
\text { behavior in adults. }\end{array}$} \\
\hline & $\begin{array}{l}\text { - Suicidal ideation and behavior in adults } \\
\text { - Suicidal behavior in depression }\end{array}$ & $\begin{array}{l}3 \\
3\end{array}$ & \\
\hline $\begin{array}{l}\text { Direct psychosocial and behavioral } \\
\text { interventions (interventions that } \\
\text { address suicidal behavior during } \\
\text { treatment) }\end{array}$ & Suicide attempts ${ }^{24}$ & 3 & \multirow[t]{2}{*}{$\begin{array}{l}\text { Indirect interventions did not reach } \\
\text { statistical significance. }\end{array}$} \\
\hline Dialectical behavior therapy & $\begin{array}{l}\text { - Suicide attempts and self-harm in } \\
\text { adolescents } \\
\text { - } \quad \text { Borderline personality disorder }^{25}\end{array}$ & $\begin{array}{l}3 \\
2\end{array}$ & \\
\hline Interpersonal therapy & $\begin{array}{l}\text { - Nonpsychotic major depression }{ }^{26} \\
\text { - } \quad \text { Depressed adolescents }\end{array}$ & $\begin{array}{l}3 \\
4\end{array}$ & \multirow[t]{2}{*}{$\begin{array}{l}\text { SSRIs are superior to interpersonal } \\
\text { therapy. }\end{array}$} \\
\hline Mentalization-based therapy & $\begin{array}{l}\text { - Suicide attempts and self-harm in } \\
\text { adolescents }{ }^{21} \\
\text { - } \quad \text { Borderline personality disorder }{ }^{12}\end{array}$ & $\begin{array}{l}3 \\
5\end{array}$ & \\
\hline Psychodynamic approaches & Borderline personality disorder ${ }^{25}$ & 2 & \\
\hline
\end{tabular}

SSRI = selective serotonin reuptake inhibitor. 
not provide scientific evidence that offering more psychoeducational strategies after self-injurious behaviors has a significant effect on the risk of subsequent suicide. ${ }^{14}$ Therefore, one cannot start from the assumption that psychotherapeutic techniques alone will be effective for all patients with suicidal behavior.

Focal psychotherapies have become truly relevant in terms of yielding positive results in mental disorders. In most evidence-based psychotherapeutic techniques to prevent suicide, there is a commitment to intervening on behavioral distortions and dysfunctional cognitions and emotions through a systematic procedure focused on specific goals. Techniques aimed at cognitive-behavioral models form the basis of most studies that have provide evidenced of a reduced risk of suicide. ${ }^{12}$

Calati \& Courtet evaluated patients who received psychotherapy for suicidal behavior due to schizophrenia and mood disorders. The authors noted that psychotherapy patients during follow-up were less likely to attempt suicide, resulting in an estimated number-needed-to-treat of $15 .^{20}$

Meerwijk et al. ${ }^{24}$ compared indirect interventions aimed at symptoms associated only with suicide (e.g., quality of life, anxiety, despair and depression) with interventions focused on suicidal behavior, including suicidal ideation, during treatment (direct interventions). In comparisons between direct and indirect interventions, the results after treatment did not reach statistical significance, and demonstrated a large effect size (Cohen's $d=0.97$ ). The difference was not significant on long-term follow-up, but still had a representative effect size (Cohen's $d=0.47$ ). The clinical relevance of direct interventions was emphasized by this effect size.

Cuijpers et al., ${ }^{28}$ in a meta-analysis that included 13 studies with 616 patients, analyzed the effect of psychotherapeutic interventions for depression on hopelessness, depression, and the risk of suicide. The results of three studies of psychotherapeutic interventions in depression associated with suicide risk and suicidal ideation were also included. The meta-analysis found no statistically significant effects of psychotherapy on suicidal ideation, and suicide risk was small $(\mathrm{g}=0.12 ; 95 \% \mathrm{Cl} 0.20-0.44)$. Their results must be interpreted with caution, considering that cognition (such as attention bias) is a possible therapeutic target in patients with suicidal ideation. However, the suicidal phenotype is very heterogeneous, as are different cognitive functioning and personality traits. ${ }^{29}$ The inclusion of subjects with low levels of suicide risk may be another reason for the nonsignificant results of these studies. ${ }^{28}$ Future studies capable of differentiating homogeneous categories according to predictors of response to psychotherapy are necessary. ${ }^{28}$

\section{Pharmacotherapy}

Pharmacological interventions for suicidal behavior and their level of evidence are presented in Table 2. Details are discussed below.

\section{Antidepressants}

As discussed in part 1 of these guidelines, ${ }^{4}$ the risk of suicide may be increased by the use of antidepressants in the initial phase of treatment. However, this information needs to be analyzed carefully. First, there is an increased risk for untreated patients who have severe depressive symptoms. ${ }^{44}$ On the other hand, this event occurs in a small number of patients. ${ }^{45}$ Although some studies indicate a risk of suicide in certain groups, others demonstrate protection with such approaches. ${ }^{45,46}$ In the initial days of treatment, this risk is greater, making it small and liable to be minimized with educational interventions, supervision, and psychotherapy. ${ }^{46}$

Table 2 Pharmacological interventions for suicidal behavior

\begin{tabular}{|c|c|c|c|}
\hline Medication & Indication & $\begin{array}{l}\text { Level of } \\
\text { evidence }\end{array}$ & Warning \\
\hline \multicolumn{4}{|l|}{ Antidepressants } \\
\hline SSRI & Adults with depression $26,30,31$ & 1 & $\begin{array}{l}\text { Close monitoring in the first } 30 \text { days of use, } \\
\text { especially in adolescents. }\end{array}$ \\
\hline Venlafaxine & Adults and geriatric patients with depression ${ }^{30,31}$ & 1 & \\
\hline \multicolumn{4}{|l|}{ Antipsychotics } \\
\hline Aripiprazole & Depression with psychotic symptoms ${ }^{32}$ & 5 & As adjunct to antidepressants. \\
\hline Clozapine & Schizophrenia and schizophrenia-like psychoses ${ }^{33,34}$ & 1 & Compared to olanzapine and other agents. ${ }^{33,34}$ \\
\hline Olanzapine & $\begin{array}{l}\text { Schizophrenia, schizoaffective, or schizophreniform } \\
\text { disorder }\end{array}$ & 4 & \\
\hline Risperidone & $\begin{array}{l}\text { Schizophrenia, schizoaffective, or schizophreniform } \\
\text { disorder }{ }^{32}\end{array}$ & 4 & \\
\hline Quetiapine & Bipolar depression ${ }^{32}$ & 4 & As adjunct to lithium. \\
\hline \multirow[t]{3}{*}{ Ketamine } & Bipolar disorder 35,36 & 4 & Experimental. Off-label use. \\
\hline & Major depressive disorder $37-40$ & 4 & Not recommended for all cases. \\
\hline & Mood and anxiety spectrum disorders ${ }^{41}$ & 4 & No evidence of sustained improvement. \\
\hline \multirow[t]{2}{*}{ Lithium } & Bipolar disorder ${ }^{42}$ & 1 & $\begin{array}{l}\text { Therapeutic concentrations. Watch for side } \\
\text { effects. Risk of use in suicide attempt. }\end{array}$ \\
\hline & Major depressive disorder ${ }^{43}$ & & \\
\hline
\end{tabular}

SSRI = selective serotonin reuptake inhibitor . 
In a meta-analysis, fluoxetine and venlafaxine reduced long-term suicidal behaviors in geriatric and adult patients. The severity of the depressive condition, in any age group, decreased with the use of medication. Therefore, evidence for an increased risk of suicide in young patients who received the medication was inconclusive. ${ }^{4,30}$

Evidence supporting a causal relationship between suicide in children and the use of antidepressants was weak in another study. The introduction of sources of variability with potential significance and the use of sensitivity verification in assessments of the consequences of the model's fundamentals, as well as their impact on relevant regulatory decisions, were made possible by the use of Bayesian hierarchical models. ${ }^{47}$ On the other hand, the Canadian Network for Mood and Anxiety Treatments (CANMAT) 2016 report noted that observational studies with different antidepressants have not found changes in the risk of suicide. ${ }^{31}$ Suicide attempts and suicide risk almost doubled with exposure to selective serotonin reuptake inhibitors among adolescents in these observational studies (odds ratio $=1.92$ ). Bearing in mind that the possibility that only the most severely ill adolescents received the prescribed antidepressants cannot be ruled out, this observational sample may have a high risk of suicidal acts. Nevertheless, caution is required when prescribing to this age group. ${ }^{4,31}$

What we propose then is that treatment is a way to reduce the risk of suicide attempts and completed suicides; however, in the case of antidepressants, close surveillance is needed in the first 30 days, especially in youths.

\section{Lithium}

The greater efficiency of lithium over placebo in decreasing the number of suicides and deaths from any cause was demonstrated in a study by Cipriami. For prevention of self-harm (parasuicidal acts), lithium was not superior to placebo. Nevertheless, lithium has been linked to a reduction in the risk of suicide and to a lower number of deaths when compared to placebo in the treatment of unipolar depression. ${ }^{48}$

A meta-analysis by Baldessarini et al. ${ }^{42}$ of 18 months of treatment with lithium in bipolar patients found that the risks of completed suicide and suicide attempts were significantly lowered, by approximately $80 \%$. Blood lithium concentrations in long-term treatment generally ranged between 0.50 and 0.60 and $0.80-1.00 \mathrm{mEq} / \mathrm{L}$. Some patients required higher concentrations, while for others, lower concentrations were especially well tolerated. ${ }^{49}$

It is important to note that there are also negative results regarding lithium. One author noted that the World Health Organization (WHO) Brief Intervention and Contact $(\mathrm{BIC})$ was associated with significantly lower odds of suicide. On the other hand, six RCTs of lithium yielded nonsignificant findings. ${ }^{37}$ Although these are important data, the author did not comment on the differences in response to the technique and lithium when referring to different diagnoses.

\section{Ketamine}

Ketamine appeared to rapidly reduce suicidal ideation. Effect sizes were moderate to large at any time after a single dose (Cohen's $d=0.51-0.85$ ). Compared to placebo or midazolam, ketamine significantly decreased suicidality scores on the Montgomery-Åsberg Depression Rating Scale (MADRS), Hamilton Depression Rating Scale (HAM-D), and Quick Inventory of Depressive Symptomatology-Self Report (QIDS-SR) scales, even after adjustment for changes in the severity of depressive symptoms. ${ }^{50}$ Bartoli et al. reported that a single dose of ketamine in patients with mood disorders may decrease suicidal ideation; however, their analysis was restricted to single-arm studies. ${ }^{35}$

Although the effectiveness of ketamine in suicidal behavior (especially suicidal ideation) is based on some evidence, in patients with depression (unipolar or bipolar), these data are still preliminary and come from studies with a small number of patients. In addition, ketamine is still an off-label and experimental treatment. ${ }^{31,51}$ Another problem is that there is no evidence of sustained improvement with ketamine treatment; that is, it is not known for how long the response will be present. Therefore, we do not recommend the routine use of ketamine in clinical practice.

\section{Clozapine and other antipsychotics}

Clozapine has been studied for suicidal behavior in schizophrenia patients. In comparison to an olanzapine group, the clozapine group required fewer hospitalizations to avoid suicide attempts ${ }^{33}$ and schizophrenia, schizoaffective, and schizophreniform disorder symptoms were decreased when treated with clozapine compared to other agents. ${ }^{33,34}$

Other antipsychotics have weak indications for depression with psychotic symptoms, such as aripiprazole ${ }^{32}$; olanzapine and risperidone for schizophrenia, schizoaffective, or schizophreniform disorder ${ }^{32}$; and quetiapine plus lithium for bipolar depression. ${ }^{32}$

As the evidence is still contradictory, it is important to emphasize that the focus should always be on achieving remission of the symptoms of the underlying disorder, especially in those where suicidal behavior arises during an episode of exacerbation.

Drug treatment focused on protocols for mental illness should be one of the priorities in monitoring a patient with suicidal behavior. Preventive strategies are important, but they should not override the underlying disease. Therefore, it is always recommended that protocols specific for each diagnosis be used, especially for mood disorders. ${ }^{52}$

\section{Rapid tranquilization, sedation, and antianxiety agents}

Studies that assess the safety and effectiveness of sedative medications, rapid tranquilization, and even the use of anxiolytics in suicidal behavior are lacking. Therefore, each case must be evaluated separately.

Although there are no studies that indicate the practice, rapid reassurance in a hospital environment or in the emergency room should be considered for patient protection in cases of suicidal behavior associated with agitation or high risk of suicide (only in the first hours of admission). For this, previous protocols for psychomotor agitation should be used as a reference. ${ }^{53,54}$ 
As anxiety is a factor associated with suicide that can be modified, treatment may have the potential to decrease this risk. Although symptoms can be mitigated by treatment with benzodiazepines in the short term, research on the risk of suicide with antianxiety treatment is quite limited. No distinction in frequency of suicidal behavior was observed between individuals who received active agents and those who received placebo; such a measure should be considered in the case of exacerbated anxiety symptoms and insomnia. ${ }^{55}$ However, care should be taken when these medications are prescribed for outpatient use, as they can be used in suicide attempts.

\section{Neuromodulation}

Electroconvulsive therapy (ECT) is effective for severely depressed patients ${ }^{56-58}$ (Level of evidence 1). It is also recommended for bipolar disorder, ${ }^{51,59}$ schizophrenia, ${ }^{59,60}$ pregnant women $^{61,62}$ (for depression and suicide risk; for bipolar and psychotic disorders), elderly individuals with depression, ${ }^{61}$ and Parkinson's disease with depression. ${ }^{61}$

Compared with psychopharmacotherapy, ECT exerted superior anti-suicidal effects in patients with unipolar depression $^{63}$ (Level of evidence 3). Although data about the superior anti-suicidal effects of ECT in the treatment of patients with another diagnosis are lacking, ECT is an effective treatment and an option, either because of its direct effect on suicidal behavior or because of its indirect effect on the symptoms of the disease (Level of evidence 3).

Repetitive transcranial magnetic stimulation (rTMS) is inferior to ECT for depression without or with suicidal behavior; however, it has potential and some efficacy ${ }^{69}$ (Level of evidence 3). Except for evidence for the treatment of depressive symptoms, ${ }^{58}$ there are no specific data for treatment of suicidal behavior by transcranial direct current stimulation (tDCS), magnetic seizure therapy (MST), vagus nerve stimulation (VNS), or deep brain stimulation (DBS).

\section{Postvention}

A postvention is an intervention conducted after a suicide, or how clinicians manage the postsuicide aftermath. It is known to strengthen suicide prevention, destigmatize the tragedy, operationalize the confusing aftermath, and promote caregiver recovery. ${ }^{70}$ However, studies regarding its efficacy are minimal. Regarding the effectiveness of postventions, there is little evidence, since there are many differences in terms, models, levels, and focus. Therefore, in Box 2 we present some suggestions without presenting a level of evidence.

Regarding models, there are reports on school intervention $^{71}$ and two intensive grief psychotherapy programs, ${ }^{72,73}$ including complicated mourning. ${ }^{73}$ The school intervention $^{71}$ and an online support forum ${ }^{74}$ were associated with improvement in mental health complaints. In another study, a reduction in suicide through community intervention and through an intensive grief therapy program was reported. ${ }^{73}$
Box 2 Postvention: what to do

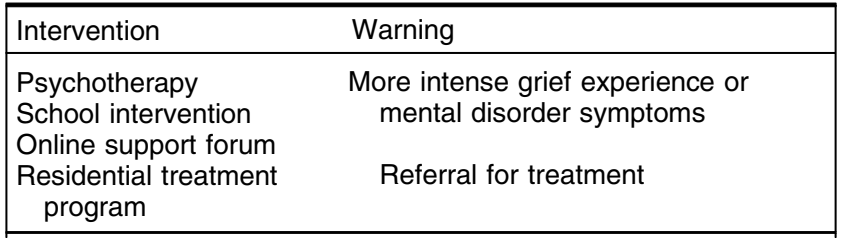

Intensive support can be provided to mourners experiencing high levels of grief or symptoms that suggest decompensated mental illness. Cha et al. ${ }^{71}$ distinguished educational support for everyone in mourning and a psychotherapeutic approach reserved for those with more intense symptoms related to grief.

Visser et al. ${ }^{75}$ differentiated the proposal to provide early support to all individuals bereaved by suicide versus referral for treatment as needed. On the other hand, Supiano et al. ${ }^{72}$ and Zisook et al. ${ }^{73}$ offered intensive mourning therapy to individuals with higher levels of symptoms.

Other data, however qualitative, report the use of online discussion forums ${ }^{74}$ and a community program ${ }^{76}$ warning of the importance of recognizing suffering, sharing experiences, and providing and receiving peer support. In addition, the favorable effects observed during a residential treatment may be related to the support and individual receives during their residential stay. ${ }^{77}$

Regarding the focus, some guidelines described their theoretical model of postintervention, ${ }^{78-80}$ giving importance to the notion of the continuity of survival (i.e., the care needs of bereaved individuals may differ depending on the intensity of the symptoms caused by the suicide). The U.S. national postvention guidelines ${ }^{78}$ were based on the disposition used by the U.S. National Suicide Prevention Strategy, ${ }^{81}$ comprising broad strategies. The UK Support after Suicide guidelines ${ }^{79}$ also point to the public health model developed by the UK national suicide prevention strategy. It outlines some types of assistance that are available to all those bereaved by suicide, those who need social support, those who are very affected, and those who need specific psychotherapy. The Postvention Australia Guidelines ${ }^{80}$ adopted a similar four-tier service delivery model. It is known that the number of individuals experiencing bereavement is greatest at the lowest level (i.e., those who will need general interventions) and smallest at the higher level (those who will need specialized interventions).

\section{Prevention and promotion}

As studies on this topic are few and difficult to compare, it is not yet possible to establish levels of evidence for this item. Therefore, Box 3 lists only suggestions from our expert panelists.

Pompili et al. observed that suicide communication (SC) was related to an odds ratio for suicide of 4.66 (95\% $\mathrm{Cl}$ 3.00-7.25), with satisfactory diagnostic accuracy only if groups of adolescents were removed from the sample. ${ }^{8}$ The available data suggest that SC occurs privately in half of suicide cases; however, it is possible that these data 


\begin{tabular}{l}
\hline Box 3 Prevention and promotion \\
\begin{tabular}{|ll|}
\hline Method & Warning \\
\hline Screening & $\begin{array}{c}\text { Be careful with suicide } \\
\text { communication }\end{array}$ \\
$\begin{array}{l}\text { Structural interventions } \\
\text { (e.g., barriers and safety nets) }\end{array}$ & \\
$\begin{array}{l}\text { World Health Organization Brief } \\
\text { Intervention and Contact }\end{array}$ & \\
$\begin{array}{l}\text { Interventions that encourage } \\
\text { help-seeking }\end{array}$ & \\
$\begin{array}{l}\text { Universal screening plus intervention } \\
\text { (Safety Plan) }\end{array}$ & $\begin{array}{c}\text { Requires access to a } \\
\text { psychiatrist }\end{array}$ \\
$\begin{array}{l}\text { Assessment and management of } \\
\text { substance misuse }\end{array}$ & \\
\hline
\end{tabular}
\end{tabular}

are underestimated, given the different ways of defining and detecting SC. Currently, SC appears to be insufficiently accurate in relation to subsequent suicide, although more research on the subject is needed.

Therefore, the absence and presence of verbalization of suicidal planning cannot be used as the only factor to predict a suicide event. It is necessary to rely on multiple risk and protection factors, on the patient's complete symptoms, previous history, and follow-up.

Some places become known as "hot spots" for suicide by jumping. Structural interventions (for example, barriers and safety nets) have been implemented in some of these locations. However, studies that have examined the effectiveness of these interventions have provided insufficient data. Even so, structural interventions at "critical points" seem to prevent suicide in those places. An increase in suicide was observed in nearby spots; however, there was a net total reduction in the number of jumping suicides. ${ }^{82}$

In an analysis of 18 studies, interventions that restricted access were associated with a reduction in the number of suicides, as were interventions that encouraged helpseeking. ${ }^{83}$ On the other hand, brief contact interventions (telephone contacts; emergency or crisis cards; postcard or letter contacts) did not have any significant effect on repeated self-mutilation, suicide attempts, or suicide. Therefore, they cannot yet be recommended for a wide clinical application. We recommend a more thorough assessment of their possible benefits in well-designed studies in clinical populations. ${ }^{17}$

The WHO BIC was evaluated in low- and middleincome countries as part of the Multisite Intervention Study on Suicidal Behaviors. The approach included an educational session on suicide prevention succeeded by regular contact with a trained professional (by phone or in person) for up to 18 months. There is no evidence that other complex psychosocial interventions reduced the risk of suicide. ${ }^{37}$

Universal screening plus intervention can be used for suicide prevention. Although its choice depends on the value placed on the outcome by decision makers, the results suggest that implementing such prevention can lead to significant cost savings. Active contact- and follow-up-type interventions were effective in preventing repeat suicide within 12 months. However, the effect at 24 months was not confirmed. ${ }^{84}$
Public health initiatives targeting suicide prevention require the assessment and management of substance misuse and therapeutic approaches associated with the assessment of suicidality. ${ }^{85}$ Suicidal ideation (SI) and suicide attempt (SA) were reported by 9.9 and $5.4 \%$ of the sample, respectively. The prevalence of SI and SA was 20.8 and $12.4 \%$, respectively, among individuals with alcohol use disorders (AUDs); 31.5 and 16.5\%, respectively, among cannabis users; and 40.0 and $20.8 \%$, respectively, among cocaine users. ${ }^{85}$

Last, despite the great growth of several technological resources, it has been suggested that digital interventions for the self-management of suicidal ideation or self-harm have negative or inconclusive results. ${ }^{86}$

\section{Conclusion}

In the second part of this guideline, we discussed the importance of screening, intervention, postvention, and prevention/promotion interventions in tackling suicidal behavior. Particular focus is given to universal screening plus intervention. Although further studies are still needed, there is already enough evidence to support some technical approaches for intervention, planning, and incorporation into public health policies.

\section{Disclosure}

LB has served as a speaker for Libbs and a scientific consultant for Apsen. KPB has served as a speaker for Libbs and Lundbeck; and received assistance for events from Torrent. GAR has served as a speaker for Pfizer, Lundbeck, Servier, Libbs, Takeda, FQM, and Genon, and received assistance for events from Torrent, Daichi Sankyo, Janssen, and Aché. AM has served as a consultant for Abbott, Jansen, Aché, Cristália, Libbs, Pfizer, Torrent, Eurofarma, Medley, Sanofi-Aventis, and Allergan; as a board advisor for Cristália, Jansen, and Libbs; as a speaker for Abbott, Aché, Cristália, Jansen, Libbs, Pfizer, and Eurofarma; receives copyrights from Guanabara Koogan; and received assistance for events from Eurofarma and Cristália. HC has served as a speaker for Janssen, Sanofi, Servier, and EMS; as a medical writer for EMS; and as a board advisor for Janssen. TCT has served on advisory boards for Janssen-Cilag, Lundbeck, Servier, Abbott, Medley, Takeda, SEM, and Torrent; and received grant or research support from Abbott, Libbs, Lundbeck, Medley, Aché, Torrent, Biolab Sanus, Pfizer, Servier, Apsen, and EMS. JQ has received clinical research support from LivaNova; has speaker bureau membership with Myriad Neuroscience, Janssen Pharmaceuticals, and Abbvie; is a stockholder at Instituto de Neurociências Dr. Joao Quevedo; and receives copyrights from Artmed Editora, Artmed Panamericana, and Elsevier/Academic Press. The other authors report no conflicts of interest.

\section{References}

1 Alarcao AC, Dell' Agnolo CM, Vissoci JR, Carvalho EC, Staton CA, de Andrade L, et al. Suicide mortality among youth in southern Brazil: 
a spatiotemporal evaluation of socioeconomic vulnerability. Braz $\mathrm{J}$ Psychiatry. 2020;42:46-53.

2 Maino MP, Morales S, Echavarri O, BarrosJ, García A, Moya C, et al. Suicide risk configuration system in a clustered clinical sample: a generalized linear model obtained through the LASSO technique. Braz J Psychiatry. 2019;41:112-21.

3 Fleischmann A, Bertolote JM, Wasserman D, De Leo D, Bolhari J, Botega NJ, et al. Effectiveness of brief intervention and contact for suicide attempters: a randomized controlled trial in five countries. Bull World Health Organ. 2008;86:703-9.

4 Baldaçara L, Rocha GA, Leite VDS, Porto DM, Grudtner RR, Diaz $\mathrm{AP}$, et al. Brazilian Psychiatric Association guidelines for the management of suicidal behavior. Part 1. Risk factors, protective factors, and assessment. Braz J Psychiatry. 2021;43:525-37.

5 Moher D, Liberati A, Tetzlaff J. Altman DG; PRISMA Group. Preferred reporting items for systematic reviews and meta-analyses: the PRISMA statement. PLoS Med. 2009;6:e1000097.

6 Suicide Prevention Resource Center (SPRC). Suicide screening and assessment [Internet]. 2014 Sep [cited 2020 Oct 5]. www.sprc.org/ sites/default/files/migrate/library/RS_suicide\%20screening_91814\%20 final.pdf

7 Lindh AU, Dahlin M, Beckman K, Strömsten L, Jokinen J, Wiktorsson $\mathrm{S}$, et al. A Comparison of suicide risk scales in predicting repeat suicide attempt and suicide: a clinical cohort study. J Clin Psychiatry. 2019;80:18m12707.

8 Pompili M, Murri MB, Patti S, Innamorati M, Lester D, Girardi P, et al. The communication of suicidal intentions: a meta-analysis. Psychol Med. 2016;46:2239-53.

9 Boudreaux ED, Miller I, Goldstein AB, Sullivan AF, Allen MH, Manton $A P$, et al. The emergency department safety assessment and follow-up evaluation (ED-SAFE): method and design considerations. Contemp Clin Trials. 2013;36:14-24.

10 Dunlap LJ, Orme S, Zarkin GA, Arias SA, Miller IW, Camargo CA Jr, et al. Screening and intervention for suicide prevention: a costeffectiveness analysis of the ED-SAFE interventions. Psychiatr Serv. 2019;70:1082-7

11 Stanley B, Brown GK, Currier GW, Lyons C, Chesin M, Knox KL. Brief intervention and follow-up for suicidal patients with repeat emergency department visits enhances treatment engagement. Am J Public Health. 2015;105:1570-2.

12 U. S. Departament of Veterans Affairs. VA/DoD Clinical Practice Guidelines: Assessment and management of patients at risk for suicide [Internet]. 2013 Jun [cited 2020 Oct 5]. www.healthquality.va. gov/guidelines/MH/srb/VADODCP SuicideRisk_Full.pdf

13 Malone D, Newron-Howes G, Simmonds S, Marriot S, Tyrer P. Community mental health teams (CMHTs) for people with severe mental illnesses and disordered personality. Cochrane Database Syst Rev. 2007:CD000270.

14 Crawford MJ, Thomas O, Khan N, Kulinskaya E. Psychosocial interventions following self-harm: systematic review of their efficacy in preventing suicide. Br J Psychiatry. 2007;190:11-7.

15 Dieterich M, Irving CB, Bergman $\mathrm{H}$, Khokhar MA, Park B, Marshall M. Intensive case management for severe mental illness. Cochrane Database Syst Rev. 2017;1:CD007906.

16 Milner A, Witt K, Pirkis J, Hetrick S, Robinson J, Currier D, et al. The effectiveness of suicide prevention delivered by GPs: a systematic review and meta-analysis. J Affect Disord. 2017;210:294-302.

17 Milner AJ, Carter G, Pirkis J, Robinson J, Spittal MJ. Letters, green cards, telephone calls and postcards: systematic and meta-analytic review of brief contact interventions for reducing self-harm, suicide attempts and suicide. Br J Psychiatry. 2015;206:184-90.

18 Noh D, Park YS, Oh EG. Effectiveness of telephone-delivered interventions following suicide attempts: a systematic review. Arch Psychiatr Nurs. 2016;30:114-9.

19 Wasserman D, Hoven CW, Wasserman C, Wall M, Eisenberg R, Hadlaczky G, et al. School-based suicide prevention programmes: the SEYLE cluster-randomised, controlled trial. Lancet. 2015;385: 1536-44.

20 Calati R, Courtet P. Is psychotherapy effective for reducing suicide attempt and non-suicidal self-injury rates? Meta-analysis and metaregression of literature data. J Psychiatr Res. 2016;79:8-20.

21 Ougrin D, Tranah T, Stahl D, Moran P, Asarnow JR. Therapeutic interventions for suicide attempts and self-harm in adolescents: systematic review and meta-analysis. J Am Acad Child Adolesc Psychiatry. 2015;54:97-107.e2.

22 Leavey K, Hawkins R. Is cognitive behavioural therapy effective in reducing suicidal ideation and behaviour when delivered face-to-face or via e-health? A systematic review and meta-analysis. Cogn Behav Ther. 2017;46:353-74.

23 Gotzsche PC, Gotzsche PK. Cognitive behavioural therapy halves the risk of repeated suicide attempts: systematic review. J R Soc Med. 2017;110:404-10.

24 Meerwijk EL, Parekh A, Oquendo MA, Allen IE, Franck LS, Lee KA. Direct versus indirect psychosocial and behavioural interventions to prevent suicide and suicide attempts: a systematic review and metaanalysis. Lancet Psychiatry. 2016;3:544-54.

25 Cristea IA, Gentili C, Cotet CD, Palomba D, Barbui C, Cuijpers P. Efficacy of psychotherapies for borderline personality disorder: a systematic review and meta-analysis. JAMA Psychiatry. 2017;74:319-28.

26 Rucci P, Frank E, Scocco P, Calugi S, Miniati M, Fagiolini A, et al. Treatment-emergent suicidal ideation during 4 months of acute management of unipolar major depression with SSRI pharmacotherapy or interpersonal psychotherapy in a randomized clinical trial. Depress Anxiety. 2011;28:303-9.

27 Tang TC, Jou SH, Ko CH, Huang SY, Yen CF. Randomized study of school-based intensive interpersonal psychotherapy for depressed adolescents with suicidal risk and parasuicide behaviors. Psychiatry Clin Neurosci. 2009;63:463-70.

28 Cuijpers P, de Beurs DP, van Spijker BA, Berking M, Andersson G, Kerkhof AJ. The effects of psychotherapy for adult depression on suicidality and hopelessness: a systematic review and meta-analysis. J Affect Disord. 2013;144:183-90.

29 da Silva AG, Malloy-Diniz LF, Garcia MS, Figueiredo CG, Figueiredo RN, Diaz AP, et al. Cognition as a therapeutic target in the suicidal patient approach. Front Psychiatry. 2018;9:31.

30 Gibbons RD, Brown CH, Hur K, Davis J, Mann JJ. Suicidal thoughts and behavior with antidepressant treatment: reanalysis of the randomized placebo-controlled studies of fluoxetine and venlafaxine. Arch Gen Psychiatry. 2012;69:580-7.

31 Kennedy SH, Lam RW, Mclntyre RS, Tourjman SV, Bhat V, Blier P, et al. Canadian network for mood and anxiety treatments (CANMAT) 2016 clinical guidelines for the management of adults with major depressive disorder: section 3. Pharmacological treatments. Can J Psychiatry. 2016;61:540-60.

32 Pompili M, Baldessarini RJ, Forte A, Erbuto D, Serafini G, Fiorillo A, et al. Do atypical antipsychotics have antisuicidal effects? A hypothesis-generating overview. Int J Mol Sci. 2016;17:1700.

33 Lobos CA, Komossa K, Rummel-Kluge C, Hunger H, Schmid F, Schwarz S, et al. Clozapine versus other atypical antipsychotics for schizophrenia. Cochrane Database Syst Rev.2010;(11):CD006633.

34 Hennen J, Baldessarini RJ. Suicidal risk during treatment with clozapine: a meta-analysis. Schizophr Res. 2005;73:139-45.

35 Bartoli F, Riboldi I, Crocamo C, Di Brita C, Clerici M, Carra G. Ketamine as a rapid-acting agent for suicidal ideation: a meta-analysis. Neurosci Biobehav Rev. 2017;77:232-6.

36 Grunebaum MF, Ellis SP, Keilp JG, Moitra VK, Cooper TB, Marver $\mathrm{JE}$, et al. Ketamine versus midazolam in bipolar depression with suicidal thoughts: a pilot midazolam-controlled randomized clinical trial. Bipolar Disord. 2017;19:176-83.

37 Riblet NB, Shiner B, Young-Xu Y, Watts BV. Strategies to prevent death by suicide: meta-analysis of randomised controlled trials. $\mathrm{Br} \mathrm{J}$ Psychiatry. 2017;210:396-402.

38 Canuso CM, Singh JB, Fedgchin M, Alphs L, Lane R, Lim P, et al. Efficacy and safety of intranasal esketamine for the rapid reduction of symptoms of depression and suicidality in patients at imminent risk for suicide: results of a double-blind, randomized, placebo-controlled study. Am J Psychiatry. 2018;175:620-30.

39 Chen MH, Lin WC, Wu HJ, Cheng CM, Li CT, Hong CJ, et al. Antisuicidal effect, BDNF Val66Met polymorphism, and low-dose ketamine infusion: reanalysis of adjunctive ketamine study of Taiwanese patients with treatment-resistant depression (AKSTP-TRD). J Affect Disord. 2019;251:162-9.

40 Price RB, losifescu DV, Murrough JW, Chang LC, Al Jurdi RK, Iqbal $S Z$, et al. Effects of ketamine on explicit and implicit suicidal cognition: a randomized controlled trial in treatment-resistant depression. Depress Anxiety. 2014;31:335-43. 
41 Murrough JW, Soleimani L, DeWilde KE, Collins KS, Lapidus KA, lacoviello BM, et al. Ketamine for rapid reduction of suicidal ideation: a randomized controlled trial. Psychol Med. 2015;45:3571-80.

42 Baldessarini RJ, Tondo L, Davis P, Pompili M, Goodwin FK, Hennen J. Decreased risk of suicides and attempts during long-term lithium treatment: a meta-analytic review. Bipolar Disord. 2006;8: 625-39.

43 Cipriani A, Pretty H, Hawton K, Geddes JR. Lithium in the prevention of suicidal behavior and all-cause mortality in patients with mood disorders: a systematic review of randomized trials. Am J Psychiatry. 2005;162:1805-19.

44 Dold M, Bartova L, Fugger G, Kautzky A, Souery D, Mendlewicz J, et al. Major depression and the degree of suicidality: results of the european group for the study of resistant depression (GSRD). Int J Neuropsychopharmacol. 2018;21:539-49.

45 Kaminski JA, Bschor T. Antidepressants and suicidality: a re-analysis of the re-analysis. J Affect Disord. 2020;266:95-9.

46 Brent DA. Antidepressants and suicidality. Psychiatr Clin North Am. 2016;39:503-12.

47 Kaizar EE, Greenhouse JB, Seltman H, Kelleher K. Do antidepressants cause suicidality in children? A Bayesian meta-analysis. Clin Trials. 2006;3:73-90; discussion 91-8.

48 Cipriani A, Hawton K, Stockton S, Geddes JR. Lithium in the prevention of suicide in mood disorders: updated systematic review and meta-analysis. BMJ. 2013;346:f3646.

49 Tondo L, Alda M, Bauer M, Bergink V, Grof P, Hajek T, et al. Clinical use of lithium salts: guide for users and prescribers. Int $\mathrm{J}$ Bipolar Disord. 2019;7:16.

50 Wilkinson ST, Ballard ED, Bloch MH, Mathew SJ, Murrough JW, Feder A, et al. The effect of a single dose of intravenous ketamine on suicidal ideation: a systematic review and individual participant data meta-analysis. Am J Psychiatry. 2018;175:150-8.

51 Yatham LN, Kennedy SH, Parikh SV, Schaffer A, Bond DB, Frey BN, et al. Canadian network for mood and anxiety treatments (CANMAT) and international society for bipolar disorders (ISBD) 2018 guidelines for the management of patients with bipolar disorder. Bipolar Disord. 2018;20:97-170.

52 Lima AF, Miguel SR, Cohen M, Zimmermann JJ, Shansis FM, Cruz $\mathrm{LN}$, et al. Effectiveness evaluation of mood disorder treatment algorithms in Brazilian public healthcare patients. Braz J Psychiatry. 2018;40:26-34

53 Baldacara L, Diaz AP, Leite V, Pereira LA, Dos Santos RM, Gomes Júnior VP, et al. Brazilian guidelines for the management of psychomotor agitation. Part 2. Pharmacological approach. Braz J Psychiatry. 2019;41:324-35.

54 Baldacara L, Ismael F, Leite V, Pereira LA, Dos Santos RM, Gomes Júnior VP, et al. Brazilian guidelines for the management of psychomotor agitation. Part 1. Non-pharmacological approach. Braz J Psychiatry. 2019;41:153-67.

55 Work Group on Suicidal Behaviors. Practice guideline for the assessment and treatment of patients with suicidal behaviors. Arlington: American Psychiatric Association, 2003.

$56 \mathrm{Kho} \mathrm{KH}$, van Vreeswijk MF, Simpson S, Zwinderman AH. A metaanalysis of electroconvulsive therapy efficacy in depression. J ECT. 2003;19:139-47.

57 Mochcovitch MD, Baczynski TP, Telles LL, Alves M, Rosa M, Lucca $\mathrm{G}$, et al. Eletroconvulsoterapia [Internet]. [cited 2020 Oct 6]https:// diretrizes.amb.org.br/_DIRETRIZES/eletroconvulsoterapia/files/assets/ common/downloads/publication.pdf

58 Milev RV, Giacobbe P, Kennedy SH, Blumberger DM, Daskalakis ZJ, Downar J, et al. Canadian network for mood and anxiety treatments (CANMAT) 2016 clinical guidelines for the management of adults with major depressive disorder: Section 4. Neurostimulation treatments. Can J Psychiatry. 2016;61:561-75.

59 Rosenquist PB, Youssef NA, Surya S, McCall WV. When all else fails: the use of electroconvulsive therapy for conditions other than major depressive episode. Psychiatr Clin N Am. 2018;41:355-71.

60 Zheng W, Cao XL, Ungvari GS, Xiang YQ, Guo T, Liu ZR, et al. Electroconvulsive therapy added to non-clozapine antipsychotic medication for treatment resistant schizophrenia: meta-analysis of randomized controlled trials. PLoS One. 2016;11:e0156510.

61 Hermida AP, Glass OM, Shafi H, McDonald WM. Electroconvulsive therapy in depression. current practice and future direction. Psychiatr Clin N Am. 2018;41:341-53.
62 Ward HB, Fromson JA, Cooper JJ, De Oliveira G, Almeida M. Recommendations for the use of ECT in pregnancy: literature review and proposed clinical protocol. Arch Womens Ment Health. 2018;21: 715-22.

63 Liang CS, Chung CH, Ho PS, Tsai CK, Chien WC. Superior antisuicidal effects of electroconvulsive therapy in unipolar disorder and bipolar depression. Bipolar Disord. 2018;20:539-46.

64 Methfessel I, Sartorius A, Zilles D. Electroconvulsive therapy against the patients' will: a case series. World J Biol Psychiatry. 2018;19:236-42.

65 Grover S, Sahoo S, Rabha A, Koirala R. ECT in schizophrenia: a review of the evidence. Acta Neuropsychiatr. 2019;31:115-27.

66 Phutane VH, Thirthalli J, Kesavan M, Kumar NC, Gangadhar BN. Why do we prescribe ECT to schizophrenia patients? Indian J Psychiatry. 2011;53:149-51.

67 Petrides G, Fink M, Husain MM, Knapp RG, Rush AJ, Mueller M et al. ECT remission rates in psychotic versus nonpsychotic depressed patients: a report from CORE. J ECT. 2001;17:244-53.

68 Fink M, Kellner $\mathrm{CH}$, McCall WV. The role of ECT in suicide prevention. J ECT. 2014;30:5-9.

69 Keshtkar M, Ghanizadeh A, Firoozabadi A. Repetitive transcranial magnetic stimulation versus electroconvulsive therapy for the treatment of major depressive disorder, a randomized controlled clinical trial. J ECT. 2011;27:310-4

70 Erlich MD, Rolin SA, Dixon LB, Adler DA, Oslin DW, Levine B, et al Why we need to enhance suicide postvention: evaluating a survey of psychiatrists' behaviors after the suicide of a patient. J Nerv Ment Dis. 2017;205:507-11.

71 Cha JM, Kim JE, Kim MA, Shim B, Cha MJ, Lee JJ, et al. Five months follow-up study of school-based crisis intervention for Korean high school students who experienced a peer suicide. J Korean Med Sci. 2018;33:e192.

72 Supiano KP, Haynes LB, Pond V. The transformation of the meaning of death in complicated grief group therapy for survivors of suicide: a treatment process analysis using the meaning of loss codebook. Death Stud. 2017;41:553-61.

73 Zisook S, Shear MK, Reynolds CF, Simon NM, Mauro C, Skritskaya NA, et al. Treatment of complicated grief in survivors of suicide loss: a HEAL report. J Clin Psychiatry. 2018;79:17m11592.

74 Kramer J, Boon B, Schotanus-Dijkstra M, van Ballegooijen W, Kerkhof $A$, van der Poel $A$. The mental health of visitors of web-based support forums for bereaved by suicide. Crisis. 2015;36:38-45.

75 Comans TA, Visser VS, Scuffham P. Evaluation of the effectiveness of a community-based crisis intervention program for people bereaved by suicide. J Community Psychol. 2014;42:19-28.

76 Peters K, Staines A, Cunningham C, Ramjan L. The lifekeeper memory quilt: evaluation of a suicide postvention program. Death Stud. 2015;39:353-9.

77 Scocco P, Zerbinati L, Preti A, Ferrari A, Totaro S. Mindfulnessbased weekend retreats for people bereaved by suicide (Panta Rhei): a pilot feasibility study. Psychol Psychother. 2019;92:39-56.

78 Suicide Prevention Resource Center. Responding to grief, trauma and distress after a suicide: U.S. National Guidelines [Internet]. 2015 [cited 2020 Oct 6]. www.sprc.org/resources-programs/respondinggrief-trauma-and-distress-after-suicide-us-national-guidelines

79 Public Health England. Support after a suicide: a guide to providing local services: a practice resource [Internet]. 2016 Oct [cited 2020 Oct 6]. http://assets.publishing.service.gov.uk/government/uploads/ system/uploads/attachment_data/file/590838/support_after_a_suicide. pdf

80 Australian Institute for Suicide Research and Prevention \& Postvention Australia. Postvention Australia guidelines: a resource for organisations and individuals providing services to people bereaved by suicide [Internet]. 2017 [cited 2020 Oct 6]. www.griffith.edu.au/ data/assets/pdf_file/0038/359696/Postvention_WEB.pdf

81 U.S. Surgeon General and of the National Action Alliance for Suicide Prevention. National strategy for suicide prevention: goals and objectives for action.Washington:: US Department of Health \& Human Services; 2012.

82 Pirkis J, Spittal MJ, Cox G, Robinson J, Cheung YT, Studdert D. The effectiveness of structural interventions at suicide hotspots: a metaanalysis. Int J Epidemiol. 2013;42:541-8.

83 Pirkis J, Too LS, Spittal MJ, Krysinska K, Robinson J, Cheung YT. Interventions to reduce suicides at suicide hotspots: a systematic review and meta-analysis. Lancet Psychiatry. 2015;2:994-1001. 
84 Inagaki $\mathrm{M}$, Kawashima $\mathrm{Y}$, Kawanishi $\mathrm{C}$, Yonemoto $\mathrm{N}$, Sugimoto $\mathrm{T}$, Furuno $\mathrm{T}$, et al. Interventions to prevent repeat suicidal behavior in patients admitted to an emergency department for a suicide attempt: a meta-analysis. J Affect Disord. 2015;175:66-78.

85 Abdalla RR, Miguel AC, Brietzke E, Caetano R, Laranjeira R, Madruga CS. Suicidal behavior among substance users: data from the Second Brazilian National Alcohol and Drug Survey (II BNADS). Braz J Psychiatry. 2019;41:437-40.

86 Witt K, Spittal MJ, Carter G, Pirkis J, Hetrick S, Currier D, et al Effectiveness of online and mobile telephone applications ('apps') for the self-management of suicidal ideation and self-harm: a systematic review and meta-analysis. BMC Psychiatry. 2017;17:297. 